\title{
Effect of telmisartan vs. ramipril on 'dipping' status and blood pressure variability: pooled analysis of the PRISMA studies
}

\author{
Philippe Gosse ${ }^{1}$ and Helmut Schumacher ${ }^{2}$
}

A retrospective pooled analysis of the 'Prospective, Randomized Investigation of the Safety and Efficacy of MICARDIS vs. Ramipril Using ABPM' studies conducted in Europe and South Africa (PRISMA I) and in the United States of America and Canada (PRISMA II) was carried out to investigate the effects of telmisartan and ramipril on dipper status (extreme dippers, dippers, non-dippers, risers/reverse dippers), and blood pressure (BP) variability in 1279 patients (with normal sleeping patterns and valid 24-h ambulatory BP monitoring recordings at baseline and end point). After 14 weeks' treatment, telmisartan had a greater systolic BP (SBP) reduction and higher smoothness index in all four dipper groups compared with ramipril. In addition, the tendency toward dipping was significantly higher in patients treated with) telmisartan than ramipril $(P=0.032$; odds ratio for telmisartan vs. ramipril: 1.27 (95\% confidence interval: $1.102-1.58)$ ). In patients with an early morning SBP surge $\geqslant 35 \mathrm{~mm} \mathrm{Hg}$, telmisartan treatment was associated with significantly greater reductions from baseline in the night-time low mean, early morning mean and early morning SBP surge compared with ramipril $(P=0.026, P<0.0001$ and $\boldsymbol{P}=0.0006$, respectively). In this retrospective analysis, telmisartan was shown to normalize the circadian BP pattern to a dipper profile in a larger proportion of patients than ramipril, and reduce early-morning SBP surge in high-risk patients, indicative of a cardioprotective effect. These findings need to be confirmed in long-term prospective trials and observational studies.

Hypertension Research (2014) 37, 151-157; doi:10.1038/hr.2013.121; published online 19 September 2013

Keywords: ambulatory blood pressure monitoring; dipper type; early morning blood pressure surge; ramipril; telmisartan

\section{INTRODUCTION}

Hypertension is a well-known risk factor for cardiovascular disease (CVD) that affects both microvessels and macrovessels and evidence shows that lowering blood pressure (BP) reduces the risks of CVD. ${ }^{1,2}$ Ambulatory BP monitoring (ABPM) allows a better prediction of risk than casual or office BP. This better predictive value probably stems not only from the large number of $\mathrm{BP}$ measurements obtained and the ability to avoid the 'white-coat' effect, but also from the information gained on BP variability. Two main aspects of this variability have been proved to be important in predicting CVD: loss of BP reduction during the night-time (non-dipping pattern) and excessive BP elevation in the morning. So the effects of antihypertensive treatment on BP cannot be described only in terms of average 24-h levels anymore, and the consequences on $\mathrm{BP}$ variability should also be considered. However, the aim to reduce morning rise in BP as well as to increase BP dip at night may appear, in part, contradictory. It is, therefore, important to describe $\mathrm{BP}$ change with treatment according to dipping patterns.

A previous analysis of the two 'Prospective, Randomized Investigations of the Safety and efficacy of MICARDIS vs. ramipril using
ABPM' studies conducted in Europe and South Africa (PRISMA I) and in the United States of America and Canada (PRISMA II) demonstrated that the angiotensin II $\left(\mathrm{AT}_{1}\right)$ receptor blocker, telmisartan, which has a trough-to-peak ratio of $>90 \%,{ }^{3}$ significantly reduced the mean night-time low (NTL) BP, early morning mean (EMM) BP and the early morning BP surge (EMBPS; defined as the difference between EMM and NTL) when compared with the angiotensin-converting enzyme inhibitor, ramipril. ${ }^{4}$ In this earlier analysis, a greater reduction in the EMBPS was also observed in patients with a high EMBPS and in those who were dippers at baseline. ${ }^{4}$

Following the earlier analysis, the current report presents the findings of a new retrospective pooled analysis of the PRISMA I and II studies (NCT00274612) that investigated the effects of telmisartan and ramipril on dipper status, EMBPS and BP variability following 14 weeks' treatment.

\section{MATERIALS AND METHODS}

Study design

The PRISMA I and II studies compared telmisartan $80 \mathrm{mg}$ with ramipril $10 \mathrm{mg}$ over a 14-week treatment period; all the results have been previously

${ }^{1}$ Cardiology/Hypertension Department, Saint André Hospital, University Hospital of Bordeaux, Bordeaux, France and ${ }^{2}$ Department of Medical Data Services, Boehringer Ingelheim Pharma GmbH \& Co. KG, Ingelheim, Germany

Correspondence: Dr P Gosse, Service de Cardiologie-Hypertension Arterielle, Hoŝpital Saint-André, 1 rue Jean Burguet, Bordeaux 33075, France.

E-mail: philippe.gosse@chu-bordeaux.fr

Received 18 February 2013; revised 11 June 2013; accepted 2 August 2013; published online 19 September 2013 
reported. $^{4-6}$ In brief, patients discontinued antihypertensive treatment following the screening visit in both studies and received placebo treatment for 2 weeks ( 4 weeks for those who were previously prescribed angiotensinconverting enzyme inhibitors, angiotensin II $\left(\mathrm{AT}_{1}\right)$ receptor blockers, or diuretics). Patients were then randomized to receive telmisartan $40 \mathrm{mg}$ or ramipril $2.5 \mathrm{mg}$ for 2 weeks, followed by forced titration to telmisartan $80 \mathrm{mg}$ for 12 weeks or ramipril $5 \mathrm{mg}$ for 6 weeks and $10 \mathrm{mg}$ for the remaining 6 weeks. Patients received their study medication once daily at approximately the same time each morning.

\section{Patients}

The detailed inclusion criteria for both studies have already been published. ${ }^{5,6}$ In brief, patients aged $\geqslant 18$ years with mild-to-moderate hypertension (defined as seated diastolic BP (DBP): 95-109 $\mathrm{mm} \mathrm{Hg}$ ) at enrollment, and a 24-h mean diastolic BP $\geqslant 85 \mathrm{~mm} \mathrm{Hg}$ as measured by ABPM at baseline, were included. For this current pooled analysis, patients with unusual sleep times (that is, going to bed before 1900 or after $0300 \mathrm{~h}$, waking before 0300 or after $1000 \mathrm{~h}$ ), and/or differences of sleeping duration of $>3 \mathrm{~h}$ between baseline and study end point were excluded.

\section{Patient evaluation}

The study methods have been described in detail elsewhere. ${ }^{5,6}$ In brief, changes from baseline to week 14 in 24-h BP were measured in patients using 24-h ABPM with a Spacelabs 90207 automatic ambulatory BP monitor (Spacelabs Healthcare, Issaquah, WA, USA) initiated at the time of dosing. BP was measured every $20 \mathrm{~min}$ throughout the day and night. The days for monitoring the 24-h ABPM were carefully selected to ensure that the patient's daily activities were similar on each occasion (for example, always a work day). Patients recorded their time of going to bed and of waking in a diary.

\section{Statistical analyses}

The mean $\mathrm{BP}$ was calculated over different time periods using the records from the 24-h ABPM as described previously:5,6 the 24 -h mean systolic BP (SBP) and diastolic BP were calculated using all valid measurements during the complete 24-h measurement period; sleeping mean BP was the mean BP in the period between going to bed and waking; wake mean BP was the mean BP in the period between waking and going to bed. The surge in BP was calculated on an individual patient basis as described previously: $:^{78}$ the NTL BP was the mean of all measurements within \pm 30 min around the time of the minimum BP during sleeping; EMM BP was defined as the mean during the $2 \mathrm{~h}$ after waking; and the EMBPS was the difference between the EMM and NTL. The smoothness index (SI), a measure of the homogeneity of BP reduction by antihypertensive treatment, was calculated as the mean of the hourly mean changes from baseline to study end point divided by its standard deviation. ${ }^{9}$

For the current analysis, patients were subdivided within each treatment group into four different 'dipper' categories, based on the relative reduction in sleeping mean BP vs. wake mean BP at baseline: extreme dippers (sleeping mean $\mathrm{BP} \leqslant 80 \%$ of wake mean $\mathrm{BP}$ ); dippers (sleeping mean $\mathrm{BP}>80$ to $\leqslant 90 \%$ of wake mean BP); non-dippers (sleeping BP $>90$ to $100 \%$ of wake mean $\mathrm{BP}$ ); and risers/reverse dippers (sleeping mean > wake mean $\mathrm{BP}$ ). Patients were also subdivided according to the extent of their EMBPS and further analyses were undertaken specifically on those patients with an early morning SBP surge $\geqslant 35 \mathrm{~mm} \mathrm{Hg}$ (the threshold for the upper EMBPS quartile ${ }^{4}$ was rounded to a multiple of 5)

Demographic data and baseline characteristics were compared between dipper categories using analysis of variance and the Cochran-Mantel-Haenszel test, adjusting for study.

SBP changes from baseline for the respective time intervals and the SI were analyzed for treatment differences in the 'dipper' subgroups using analysis of covariance, adjusting for baseline, study, dipper subgroup and treatment-bydipper subgroup interaction. Change in dipper status after 14 weeks' treatment was analyzed using logistic regression analysis with cumulative logits, adjusting for dipper type at baseline, study, age, gender and body mass index. A $P$-value $<0.05$ was considered statistically significant. SAS version 9.2 (SAS Institute, Cary, NC, USA) was used for the analysis.

\section{RESULTS}

\section{Patients}

A total of 1613 patients were randomized to treatment in the PRISMA I and II studies. Of these, 1279 met the criteria for normal sleeping patterns and also had valid 24-h ABPM recordings at baseline and end point. Baseline demographic and clinical characteristics for the patients stratified according to their baseline dipper category are presented in Table 1 . The majority of patients (52.9\%) were classified as dippers at baseline. Risers/reverse dippers constituted the smallest subgroup of patients $(n=52(4.1 \%))$. Male patients had a greater tendency toward dipping than female patients $(P=0.0008)$. No significant differences in terms of race were observed among the four dipper categories.

Significant differences in age $(P=0.0004)$, body weight $(P=0.038)$ and body mass index $(P=0.039)$ were observed across the four dipper subgroups after adjusting for study. In particular, patients in the riser subgroup tended to be older and have a higher body mass index than those in the other three dipper subgroups. As expected, there were significant trends across the different circadian profile subgroups in terms of mean SBP measurements and EMBPS. In particular, sleep mean SBP and NTL SBP were lowest in the extreme dipper subgroup and highest in the riser subgroup (both $P<0.0001$ ). There were also significant trends across the circadian profile subgroups in terms of the percentage of patients with morning surge ( $\geqslant 35$ and $\geqslant 55 \mathrm{~mm} \mathrm{Hg}$, both $P<0.0001$ ). The proportion of patients with morning surge $\geqslant 35$ and $\geqslant 55 \mathrm{mmHg}$ was highest in the extreme dipper subgroup $(P<0.0001)$. The riser subgroup had the lowest proportion of patients with morning surge $\geqslant 35 \mathrm{~mm} \mathrm{Hg}$ and none with morning surge $\geqslant 55 \mathrm{~mm} \mathrm{Hg}$.

\section{BP changes}

Figure 1 presents the mean 24-h SBP profiles at baseline and end point for the telmisartan and ramipril groups, stratified according to baseline dipper categories. In the telmisartan group, all four dipper subgroups showed a reduction from baseline to end of trial in SBP at each time point over the 24-h monitoring period (Figure 1a). Similar changes were observed for ramipril (Figure 1b). Changes in SBP in different time intervals during the 24-h monitoring period from baseline to the end of the trial for each dipper category are presented in Table 2 .

No significant interaction between treatment and dipping type was observed for each SBP measure for different intervals during the 24-h monitoring period, indicating that the difference between treatment groups was consistent across all dipping types (Table 2). Telmisartan significantly reduced the 24-h mean, wake mean, sleep mean, NTL mean, EMM and EMBPS across all four dipper categories compared with ramipril (all adjusted $P$-values $<0.05$ ). The SI was higher after telmisartan treatment than after ramipril treatment in each dipper subgroup (Table 2; adjusted treatment difference, $P=0.0006$ ).

A total of 386 of 1279 patients (30.2\%) had an early morning SBP surge $\geqslant 35 \mathrm{~mm} \mathrm{Hg}, 211$ in the telmisartan group and 175 in the ramipril group. SBP at baseline and end point and change in SBP from baseline to study end point for patients with an early morning SBP surge $\geqslant 35 \mathrm{mmHg}$ are presented in Table 3. Telmisartan treatment was associated with significantly greater reductions from baseline in the NTL, EMM and morning surge SBPs compared with ramipril ( $P=0.026, P<0.0001$ and $P=0.0006$, respectively).

\section{Change in dipper category}

Figure 2 shows the percentage SBP reduction during sleep time by dipper type for telmisartan and ramipril. Although the mean 
Table 1 Baseline demographic and clinical characteristics of patients stratified according to their baseline dipper category ${ }^{a}$

\begin{tabular}{|c|c|c|c|c|c|c|}
\hline \multirow[b]{2}{*}{ Characteristic } & \multicolumn{4}{|c|}{ Dipper type } & \multirow[b]{2}{*}{ Total } & \multirow[b]{2}{*}{$\mathrm{P}-$ value $^{\mathrm{b}}$} \\
\hline & Extreme dipper & Dipper & Non-dipper & Riser & & \\
\hline Female, $n(\%)$ & $50(10.5)$ & $222(46.4)$ & $179(37.4)$ & $27(5.6)$ & 478 (100.0) & 0.0008 \\
\hline Male, $n(\%)$ & $85(10.6)$ & $455(56.8)$ & $236(29.5)$ & $25(3.1)$ & $801(100.0)$ & \\
\hline Age, years, mean (s.d.) & $52.0(8.7)$ & $52.8(10.1)$ & $54.2(10.3)$ & $57.9(10.5)$ & $53.3(10.1)$ & 0.0004 \\
\hline Race, $n(\%)$ & & & & & & 0.12 \\
\hline Asian & $1(3.3)$ & 19 (63.3) & $9(30.0)$ & $1(3.3)$ & $30(100.0)$ & \\
\hline Black & $2(3.6)$ & $24(43.6)$ & $27(49.1)$ & $2(3.6)$ & $55(100.0)$ & \\
\hline Caucasian & $117(11.0)$ & $564(53.2)$ & $335(31.6)$ & $45(4.2)$ & $1061(100.0)$ & \\
\hline Not available & $15(11.3)$ & $70(52.6)$ & $44(33.1)$ & $4(3.0)$ & $133(100.0)$ & \\
\hline Alcohol consumption history, $n$ (\%) & & & & & & 0.0003 \\
\hline Never smoked & $51(7.9)$ & $350(53.9)$ & $216(33.3)$ & $32(4.9)$ & $649(100.0)$ & \\
\hline Smoker & $38(17.7)$ & $97(45.1)$ & $74(34.4)$ & $6(2.8)$ & $215(100.0)$ & \\
\hline Trough cuff mean SBP, mean (s.d.) & $155.0(12.8)$ & $155.2(12.5)$ & $156.0(12.2)$ & $159.0(12.4)$ & $155.6(12.4)$ & 0.12 \\
\hline 24 h-mean SBP, mean (s.d.) & $142.4(11.4)$ & $146.9(11.2)$ & $148.6(11.7)$ & $153.3(13.0)$ & $147.2(11.6)$ & $<0.0001$ \\
\hline Wake mean SBP, mean (s.d.) & $154.3(12.2)$ & $154.4(11.8)$ & $152.0(11.9)$ & $151.5(13.1)$ & $153.5(11.9)$ & 0.0067 \\
\hline Sleep mean SBP, mean (s.d.) & $118.6(9.7)$ & $132.1(10.6)$ & $142.0(11.7)$ & $156.9(14.1)$ & $134.9(13.8)$ & $<0.0001$ \\
\hline Nighttime low SBP, mean (s.d.) & $107.6(9.8)$ & $121.5(11.0)$ & $131.0(12.4)$ & $144.6(15.9)$ & $124.1(14.1)$ & $<0.0001$ \\
\hline Early morning mean SBP, mean (s.d.) & $150.1(14.4)$ & $152.7(14.5)$ & $153.2(14.0)$ & $154.8(15.3)$ & $152.7(14.4)$ & 0.084 \\
\hline Morning surge SBP, mean (s.d.) & $42.5(10.6)$ & $31.3(11.5)$ & $22.3(11.1)$ & $10.3(12.3)$ & $28.7(13.4)$ & $<0.0001$ \\
\hline \multicolumn{7}{|c|}{ Morning surge SBP category, $\mathrm{n}(\%), \mathrm{mm} \mathrm{Hg}$} \\
\hline$<35$ & $34(25.2)$ & $445(65.7)$ & $366(88.2)$ & $50(96.2)$ & $895(70.0)$ & \\
\hline
\end{tabular}

Abbreviations: BMI, body mass index; BP, blood pressure; SBP, systolic blood pressure.

aFor demographic characteristics, study population percentages are calculated across the dipper categories. For the morning SBP characteristics, percentages are calculated within each dipper category. bStatistical analyses to identify trends across the dipper types were undertaken using the Cochran-Mantel-Haenszel $\chi^{2}$-test or analysis of variance, adjusted for the study.

percentage reduction in SBP during sleep time was reduced in extreme dipper and dipper groups following treatment, it increased in the non-dipper group and riser group. The mean percentage reduction in SBP at the end of 14 weeks in the extreme dipper group was $16.5 \%$ in the telmisartan group and $17.6 \%$ in the ramipril group compared with $22.8 \%$ and $23.5 \%$, respectively, at baseline.

Table 4 details the dipper type following 14 weeks' treatment. The change in dipper status after 14 weeks' treatment revealed a significant treatment effect in favor of telmisartan $(P=0.041)$. The odds ratio for telmisartan $v$ s. ramipril was 1.27 (95\% confidence interval, 1.02-1.58), indicating that there was a stronger tendency toward dipping in patients treated with telmisartan compared with ramipril. This finding was supported by a sensitivity analysis using the KruskalWallis test $(P=0.031)$.

\section{DISCUSSION}

Telmisartan shows greater SBP reduction and higher SI in all four dipper subgroups

In this retrospective analysis of data pooled from the two PRISMA studies, ${ }^{5,6}$ a comparison of the changes from baseline in SBP at different intervals during the 24 -h monitoring period demonstrated that telmisartan resulted in significantly greater SBP reductions in all four dipper subgroups compared with ramipril. Telmisartan treatment was also associated with a greater reduction in the EMBPS in the extreme dipper and dipper subgroups, a similar change in the non-dipper subgroup and a lower SBP rise in the riser subgroup compared with ramipril.

The SI, a measure of the homogeneity of $\mathrm{BP}$ reduction by antihypertensive treatment, was significantly higher in each dipper subgroup in patients treated with telmisartan compared with ramipril. The SI was $\geqslant 1$ in the extreme dippers, dippers and non-dipper subgroups of patients treated with telmisartan. Although the SI was $<1$ in the riser subgroup following telmisartan treatment, it was higher than that observed for ramipril ( 0.89 vs. 0.74 , respectively). As a high SI $(\geqslant 1)$ has been reported to be associated with positive changes in carotid artery wall thickness, ${ }^{10}$ the higher SI demonstrated by the long-acting drug, telmisartan, in this current analysis may provide some cardiovascular $(\mathrm{CV})$ benefit. For the extreme dippers, non-dippers and riser subgroups, who are already at a higher risk of CVD, a more homogenous BP might help to reduce their risks.

\section{Change of dipping pattern on treatment}

Classification of patients based on dipping pattern is poorly reproducible and this limits the usefulness of this concept in treating single 

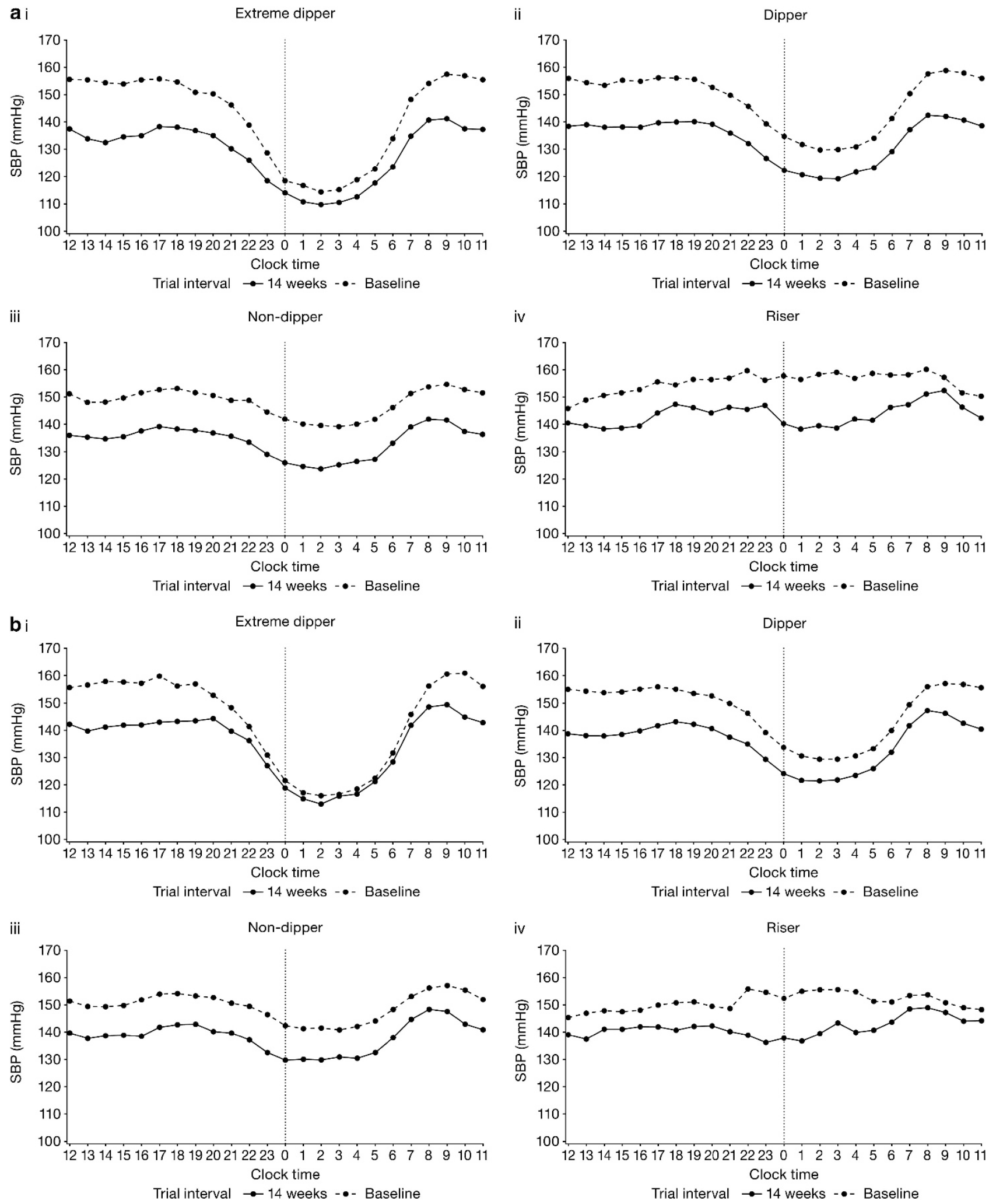

Figure 1 Mean 24-h systolic blood pressure (SBP) as measured by ambulatory blood pressure monitoring (ABPM) of patients treated with: (a) telmisartan and (b) ramipril, stratified according to their baseline dipper category: baseline and week 14 data for (i) extreme dippers, (ii) dippers, (iii) non-dippers and (iv) risers/reverse dippers. 
Table 2 Change in SBP at different time points during the 24-h monitoring period from baseline to study end point and the SI for patients treated with telmisartan $(n=634)$ or ramipril $(n=645)$ stratified according to their baseline dipper category

\begin{tabular}{|c|c|c|c|c|c|c|c|c|c|c|c|}
\hline \multirow{3}{*}{$\begin{array}{l}\text { SBP change } \\
\mathrm{mm} \mathrm{Hg}\end{array}$} & \multicolumn{8}{|c|}{ Dipper type } & \multirow{3}{*}{$\begin{array}{c}\text { Test on treatment-by- } \\
\text { dipper type interaction } \\
\text { P-value }\end{array}$} & \multirow{2}{*}{\multicolumn{2}{|c|}{$\begin{array}{c}\text { Adjusted }^{\text {a }} \text { treatment } \\
\text { difference }\end{array}$}} \\
\hline & \multicolumn{2}{|c|}{ Extreme dipper } & \multicolumn{2}{|c|}{ Dipper } & \multicolumn{2}{|c|}{ Non-dipper } & \multicolumn{2}{|c|}{ Riser } & & & \\
\hline & Telmisartan & Ramipril & Telmisartan & Ramipril & Telmisartan & Ramipril & Telmisartan & Ramipril & & Mean $(95 \% \mathrm{Cl})$ & $P$-value \\
\hline & $n=76$ & $n=59$ & $n=323$ & $n=354$ & $n=206$ & $n=209$ & $n=29$ & $n=23$ & & $645 / 634$ & \\
\hline 24-h Mean & $-13.5(9.4)$ & $-8.3(8.8)$ & $-14.2(11.0)$ & $-11.5(9.5)$ & $-14.3(11.0)$ & $-11.3(9.8)$ & $-12.1(11.4)$ & $-9.5(9.6)$ & 0.45 & $3.2(1.5-4.9)$ & 0.0002 \\
\hline Wake mean & $-17.3(11.2)$ & $-12.1(9.6)$ & $-15.7(11.8)$ & $-12.9(10.7)$ & $-13.6(11.7)$ & $-11.2(10.4)$ & $-9.1(11.3)$ & $-6.4(10.0)$ & 0.48 & $3.2(1.3-5.0)$ & 0.0008 \\
\hline Sleep mean & $-5.1(8.7)$ & $-1.0(10.0)$ & $-11.2(11.8)$ & $-8.1(9.9)$ & $-15.5(12.1)$ & $-11.4(11.3)$ & $-17.4(13.9)$ & -15.6 (11.9) & 0.60 & $3.1(1.3-5.0)$ & 0.0011 \\
\hline NTL & $-3.0(10.3)$ & $-1.2(12.2)$ & $-10.2(12.5)$ & $-7.8(11.6)$ & $-14.0(14.7)$ & $-10.7(13.5)$ & $-18.6(16.0)$ & $-15.0(13.8)$ & 0.73 & $2.5(0.4-4.6)$ & 0.0175 \\
\hline EMM & $-12.4(13.6)$ & $-5.0(10.9)$ & $-13.9(14.8)$ & $-8.4(12.0)$ & $-11.3(13.8)$ & $-8.6(11.9)$ & $-8.6(15.2)$ & $-2.0(14.8)$ & 0.36 & $5.2(3.0-7.3)$ & $<0.0001$ \\
\hline EMBPS & $-9.3(14.7)$ & $-3.6(13.4)$ & $-3.7(13.8)$ & $-0.6(12.8)$ & $2.7(15.4)$ & $2.2(14.0)$ & $10.0(13.4)$ & $12.8(15.3)$ & 0.064 & $2.5(1.5-4.5)$ & 0.015 \\
\hline SI & $1.02(0.72)$ & $0.65(0.67)$ & $1.25(0.97)$ & $0.96(0.82)$ & $1.29(1.02)$ & $1.00(0.88)$ & $0.89(0.91)$ & $0.74(0.75)$ & 0.82 & $0.27(0.12-0.42)$ & 0.0006 \\
\hline
\end{tabular}

Abbreviations: $\mathrm{Cl}$, confidence interval; EMBPS, early morning blood pressure surge; EMM, early morning mean; NTL, night-time low; SBP, systolic blood pressure; SI, smoothness index. Data presented as mean (s.d.).

adjusted for baseline, study, dipper type and treatment-by-dipper type interaction.

Table 3 SBP at baseline and change from baseline to study end point for patients with an early morning SBP surge $\geqslant 35 \mathrm{~mm}$ Hg treated with telmisartan or ramipril

\begin{tabular}{|c|c|c|c|c|c|c|c|}
\hline \multirow[b]{2}{*}{$\begin{array}{l}S B P, \\
\mathrm{~mm} \mathrm{Hg}\end{array}$} & \multicolumn{3}{|c|}{ Telmisartan $(\mathrm{n}=211)$} & \multicolumn{3}{|c|}{ Ramipril $(\mathrm{n}=175)$} & \multirow[b]{2}{*}{ P-value } \\
\hline & $\begin{array}{l}\text { Baseline, mean } \\
\text { (s.d.) }\end{array}$ & $\begin{array}{l}\text { End point, mean } \\
\text { (s.d.) }\end{array}$ & $\begin{array}{c}\text { Change from baseline } e^{\mathrm{a}} \text {, mean } \\
\text { (s.e.) }\end{array}$ & $\begin{array}{l}\text { Baseline, mean } \\
\text { (s.d.) }\end{array}$ & $\begin{array}{l}\text { End point, mean } \\
\text { (s.d.) }\end{array}$ & $\begin{array}{c}\text { Change from baseline }{ }^{a} \text {, mean } \\
\text { (s.e.) }\end{array}$ & \\
\hline NTL & $116.9(12.7)$ & $111.1(15.1)$ & $-5.8(0.85)$ & $116.9(13.4)$ & $113.8(16.6)$ & $-3.0(0.93)$ & 0.026 \\
\hline EMM & $161.1(13.5)$ & $143.6(17.2)$ & $-17.3(0.93)$ & $161.1(14.6)$ & $150.7(17.9)$ & $-10.2(1.02)$ & $<0.0001$ \\
\hline EMBPS & $44.2(7.4)$ & $32.6(12.4)$ & $-11.6(0.86)$ & $44.3(7.5)$ & $37.0(13.2)$ & $-7.2(0.95)$ & 0.0006 \\
\hline
\end{tabular}

Abbreviations: EMBPS early morning blood pressure surge; EMM, early morning mean; NTL, night-time low; SBP, systolic blood pressure.

Data presented as mean (s.d.) except for adjusted SBP change from baseline to end point, which are shown as mean (s.e.).

aAdjusted for baseline and study.

patients. In an observational study of 414 untreated hypertensive patients, it was seen that in a quarter of patients, the initial dipping pattern was changed on repeat ABPM assessment after 4 weeks. The change was more prominent in the extreme dipper and non-dipper subgroups. ${ }^{11}$ In another observational study of hypertensive and normotensive patients over a period of 1 year, $44 \%$ of patients had variable dipping status, with normal night-time BP in patients who perceived their sleep quality to be good. ${ }^{12}$ Similarly, in the Study on Ambulatory Monitoring of Pressure and Lisinopril Evaluation (SAMPLE) study, variability in dipper status was observed in $35-40 \%$ of patients with essential hypertension and left ventricular hypertrophy. ${ }^{13}$ These results highlight significant intra-subject variability in the diurnal fluctuations in ambulatory BP and dipper status, suggesting that assessments based on a single ABPM cannot be taken as independent predictors of increased CV risk. Repeated ABPMs were better able to predict an 'abnormal' nocturnal profile correctly as a part of the risk stratification. ${ }^{14}$

However, in spite of limitations related to intra-subject dipping status variability, it has been shown that abnormal circadian patterns of $\mathrm{BP}$, especially nocturnal hypertension or non-dipper status, is associated with CVD risk. ${ }^{15}$ The Coronary Artery Risk Development in Young Adults (CARDIA) study demonstrated that failure of the night-time SBP to dip sufficiently, or excessive dipping of the nighttime SBP, is associated with the presence of coronary artery calcium 10-15 years later. ${ }^{16}$ A study of 900 middle-aged men and women demonstrated that those with a non-dipping nocturnal BP profile had an increased mean intima-media thickness in the carotid artery
$(P<0.01)$, an indication of early atherosclerosis. ${ }^{17}$ The reversedipping (riser) pattern of nocturnal BP is also an independent risk factor for CVD. ${ }^{18-20}$ A meta-analysis of 3648 patients from four prospective studies revealed that the dipping pattern and the night/ day BP ratio significantly and independently predicted mortality and $\mathrm{CV}$ events in hypertensive patients without a history of major $\mathrm{CV}$ disease; the incidence of CV events was highest in patients in the riser (or reverse dipper) category. ${ }^{21}$

In the prospective, observational Progetto Ipertensione Umbria Monitoraggio Ambulatoriale (PIUMA) study, the risk of CV events was significantly increased in non-dippers and reverse dippers among 3012 initially untreated hypertension patients followed up over a period of 8.44 years. ${ }^{22}$ Similarly, in a cross-sectional study of 376 patients with hypertension, left and right ventricular structure and diastolic function were found to be significantly more impaired with the non-dippers and the reverse dippers compared with others, ${ }^{23}$ and pulse wave velocity was significantly higher in reverse dippers compared with dippers and non-dippers in untreated patients with hypertension. ${ }^{24}$ Hence, it would be highly undesirable for an antihypertensive agent to cause patients to move from the dipper category to any of the other three categories.

This retrospective analysis showed a significant treatment effect on dipper status in favor of telmisartan compared with ramipril. The odds ratio, and sensitivity analysis, indicated a stronger tendency toward dipping in patients treated with telmisartan compared with ramipril. This is expected to protect patients on telmisartan from CVD. The greater tendency for dipping observed with telmisartan $v s$. 


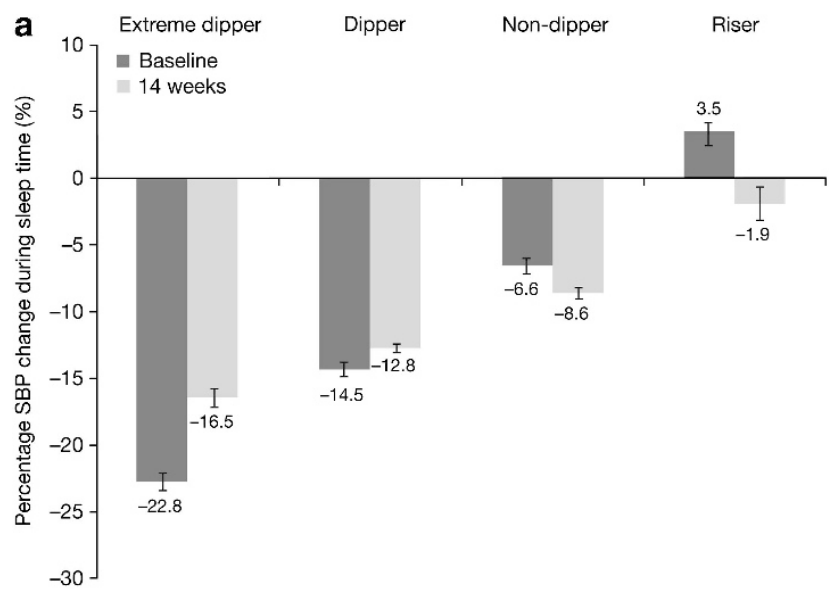

ramipril is predominantly driven by the smaller proportion at the end point of non-dippers and risers/reverse dippers with telmisartan treatment than with ramipril treatment. Transition between dipper categories following treatment might be expected due to a greater efficacy of the antihypertensive drug on the daytime BP compared with the night-time BP (resulting from a $<24 \mathrm{~h}$ duration of effect), which would make the difference between the wake and sleep BPs smaller; thus, the patient's dipper category would change, possibly from dipper to non-dipper or riser. However, telmisartan, a long-acting drug with a high trough:peak ratio, has greater efficacy on early morning $\mathrm{BP}$ (falling into the last $6 \mathrm{~h}$ of the dosing interval) compared with ramipril, ${ }^{5,6}$ which may help to maintain normal dipping status or improve the dipping status to normal. Indeed, in this analysis, only $0.9 \%$ of patients treated with telmisartan moved from the dipper to riser group, compared with $3.7 \%$ treated with ramipril. Similarly, more patients on telmisartan treatment improved from non-dipper category at baseline to dipper category at end point. Of the risers/reverse dippers at baseline, more patients on ramipril than telmisartan remained in the same category at end point. Similar proportions of patients on either treatment improved from extreme dipper category at baseline to dipper category at end point. Consistent with this finding, the mean percentage change from baseline to end point in SBP during sleep time also decreased in the extreme dipper category on either treatment.

The extreme dipper profile is commonly associated with a larger early morning SBP surge. Importantly, telmisartan treatment was associated with significantly greater reductions from baseline in the NTL, EMM, and morning surge SBPs compared with ramipril in patients with an early morning SBP surge $\geqslant 35 \mathrm{~mm} \mathrm{Hg}$, suggesting that it provides more effective BP control in these high-risk patients. A very high or very low EMBPS is reported to be associated with $\mathrm{CV}$ events. A meta-analysis of individual data from more than 5000 patients randomly recruited from eight different countries and followed up for an average of 10 years showed an EMBPS $\geqslant 37.0$ $\mathrm{mm} \mathrm{Hg}$ to be a significant and independent predictor of mortality and $\mathrm{CV}$ events, ${ }^{25}$ while in normotensive and well-controlled hypertensive elderly followed up for a period of 5 years, an EMBPS $\geqslant 34 \mathrm{~mm} \mathrm{Hg}$ was found to be associated with CV events. ${ }^{26}$ Further studies also show that those individuals who experience a high EMBPS have a higher incidence of cerebrovascular events and higher CV mortality. ${ }^{8,27}$ In contrast, in a recent study in 3012 initially untreated white patients with essential hypertension followed up for a period of 8.44 years, a blunted EMBPS $(\leqslant 9.5 \mathrm{~mm} \mathrm{Hg})$ was found to be an independent predictor of CV events. ${ }^{22}$

Ideally, antihypertensive agents should provide smooth and sustained BP control over the full 24 -h circadian cycle. ${ }^{28}$ Maintaining efficacy during the trough period can be difficult as many antihypertensive agents are taken once daily in the morning, so a longer duration of action would be expected to provide benefits in terms of reducing the EMBPS. ${ }^{29}$ Telmisartan, with its long duration of action, may help to maintain normal dipping status or improve the dipping status to normal, and to reduce BP variability during the early morning hours, the critical period of the morning surge.

In conclusion, studies have shown that BP variability adds to the prediction of $\mathrm{CV}$ events beyond average BP levels. This seems especially true for dipping pattern and the EMBPS, which means that the analysis of the effects of antihypertensive treatment can no longer be limited to average BP levels. In this retrospective analysis of two studies of identical design, telmisartan normalizes the circadian BP pattern to a dipper profile in a larger proportion of patients than ramipril and reduces EMBPS in high-risk patients. This can be expected to reduce the incidence of $\mathrm{CV}$ events, although a potential 
benefit of improved BP variability beyond average BP lowering may take longer to materialize. Further evidence is needed from long-term prospective trials and observational studies on the potential benefits of a normalized circadian profile on CV outcomes.

\section{CONFLICT OF INTEREST}

Dr Schumacher is an employee of Boehringer Ingelheim Pharma GmbH \& Co. KG. Dr Gosse has no conflict of interest regarding this work.

\section{ACKNOWLEDGEMENTS}

We were fully responsible for all content and editorial decisions, were involved at all stages of manuscript development and have approved the final version. Medical writing assistance, supported financially by Boehringer Ingelheim International $\mathrm{GmbH}$, was provided by Aruna Jeans, $\mathrm{PhD}$, of PAREXEL during the preparation of this article. We meet criteria for authorship as recommended by the International Committee of Medical Journal Editors (ICMJE) and received no compensation related to the development of the manuscript. The PRISMA studies and the pooled analysis were sponsored by Boehringer Ingelheim Pharma GmbH \& Co. KG.

1 Lewington S, Clarke R, Qizilbash N, Peto R, Collins R. Age-specific relevance of usual blood pressure to vascular mortality: a meta-analysis of individual data for one million adults in 61 prospective studies. Lancet 2002; 360: 1903-1913.

2 Staessen JA, Li Y, Thijs L, Wang JG. Blood pressure reduction and cardiovascular prevention: an update including the 2003-2004 secondary prevention trials. Hypertens Res 2005: 28: 385-407.

3 Neutel JM. Ambulatory blood pressure monitoring to assess the comparative efficacy and duration of action of a novel new angiotensin II receptor blocker-telmisartan. Blood Press Suppl 2001; 1: 27-32.

4 Gosse P, Neutel JM, Schumacher H, Lacourciere Y, Williams B, Davidai G. The effect of telmisartan and ramipril on early morning blood pressure surge: a pooled analysis of two randomized clinical trials. Blood Press Monit 2007; 12: 141-147.

5 Lacourciere Y, Neutel JM, Davidai G, Koval S. A multicenter, 14-week study of telmisartan and ramipril in patients with mild-to-moderate hypertension using ambulatory blood pressure monitoring. Am J Hypertens 2006; 19: 104-112.

6 Williams B, Gosse P, Lowe L, Harper R. The prospective, randomized investigation of the safety and efficacy of telmisartan versus ramipril using ambulatory blood pressure monitoring (PRISMA I). J Hypertens 2006; 24: 193-200.

7 Eguchi K, Kario K, Shimada K. Comparison of candesartan with lisinopril on ambulatory blood pressure and morning surge in patients with systemic hypertension. Am J Cardiol 2003; 92: 621-624.

8 Kario K, Pickering TG, Umeda Y, Hoshide S, Hoshide Y, Morinari M, Murata M, Kuroda T, Schwartz JE, Shimada K. Morning surge in blood pressure as a predictor of silent and clinical cerebrovascular disease in elderly hypertensives: a prospective study. Circulation 2003; 107: 1401-1406.

9 Parati G, Omboni S, Rizzoni D, Agabiti-Rosei E, Mancia G. The smoothness index: new, reproducible and clinically relevant measure of the homogeneity of the blood pressure reduction with treatment for hypertension. J Hypertens 1998; 16 1685-1691.

10 Rizzoni D, Muiesan ML, Salvetti M, Castellano M, Bettoni G, Monteduro C, Corbellini C, Porteri E, Guelfi D, Rosei EA. The smoothness index, but not the trough-to-peak ratio predicts changes in carotid artery wall thickness during antihypertensive treatment. J Hypertens 2001; 19: 703-711.
11 Cuspidi C, Meani S, Salerno M, Valerio C, Fusi V, Severgnini B, Lonati L, Magrini F, Zanchetti A. Reproducibility of nocturnal blood pressure fall in early phases of untreated essential hypertension: a prospective observational study. J Hum Hypertens 2004; 18: 503-509.

12 Manning G, Rushton L, Donnelly R, Millar-Craig MW. Variability of diurnal changes in ambulatory blood pressure and nocturnal dipping status in untreated hypertensive and normotensive subjects. Am J Hypertens 2000; 13: 1035-1038.

13 Omboni S, Parati G, Palatini P, Vanasia A, Muiesan ML, Cuspidi C, Mancia G. Reproducibility and clinical value of nocturnal hypotension: prospective evidence from the SAMPLE study. Study on Ambulatory Monitoring of Pressure and Lisinopril Evaluation. J Hypertens 1998; 16: 733-738.

14 Cuspidi C, Michev I, Meani S, Severgnini B, Fusi V, Corti C, Salerno M, Valerio C, Magrini F, Zanchetti A. Reduced nocturnal fall in blood pressure, assessed by two ambulatory blood pressure monitorings and cardiac alterations in early phases of untreated essential hypertension. J Hum Hypertens 2003; 17: 245-251.

15 Friedman O, Logan AG. Can nocturnal hypertension predict cardiovascular risk? Integr Blood Press Control 2009; 2: 25-37.

16 Viera AJ, Lin FC, Hinderliter AL, Shimbo D, Person SD, Pletcher MJ, Jacobs DR Jr. Nighttime blood pressure dipping in young adults and coronary artery calcium 10-15 years later: the coronary artery risk development in young adults study. Hypertension 2012; 59: 1157-1163.

17 Vasunta RL, Kesaniemi YA, Ylitalo A, Ukkola O. Nondipping pattern and carotid atherosclerosis in a middle-aged population: OPERA Study. Am J Hypertens 2012; 25: 60-66.

18 Ohkubo T, Imai Y, Tsuji I, Nagai K, Watanabe N, Minami N, Kato J, Kikuchi N, Nishiyama A, Aihara A, Sekino M, Satoh H, Hisamichi S. Relation between nocturnal decline in blood pressure and mortality. The Ohasama Study. Am J Hypertens 1997; 10: 1201-1207.

19 Verdecchia P, Porcellati C, Schillaci G, Borgioni C, Ciucci A, Battistelli M, Guerrieri M, Gatteschi C, Zampi I, Santucci A. Ambulatory blood pressure. An independent predictor of prognosis in essential hypertension. Hypertension 1994; 24: 793-801.

20 Verdecchia P, Schillaci G, Borgioni C, Ciucci A, Gattobigio R, Guerrieri M, Comparato E, Benemio G, Porcellati C. Altered circadian blood pressure profile and prognosis. Blood Press Monit 1997; 2: 347-352.

21 Fagard RH, Thijs L, Staessen JA, Clement DL, De Buyzere ML, De Bacquer DA. Nightday blood pressure ratio and dipping pattern as predictors of death and cardiovascular events in hypertension. J Hum Hypertens 2009; 23: 645-653.

22 Verdecchia P, Angeli F, Mazzotta G, Garofoli M, Ramundo E, Gentile G, Ambrosio G, Reboldi G. Day-night dip and early-morning surge in blood pressure in hypertension: prognostic implications. Hypertension 2012; 60: 34-42.

23 Ivanovic BA, Tadic MV, Celic VP. To dip or not to dip? The unique relationship between different blood pressure patterns and cardiac function and structure. J Hum Hypertens 2011; 27: 62-70.

24 Jerrard-Dunne P, Mahmud A, Feely J. Circadian blood pressure variation: relationship between dipper status and measures of arterial stiffness. J Hypertens 2007; 25 : 1233-1239.

25 Li Y, Thijs L, Hansen TW, Kikuya M, Boggia J, Richart T, Metoki H, Ohkubo T, TorpPedersen C, Kuznetsova T, Stolarz-Skrzypek K, Tikhonoff V, Malyutina S, Casiglia E, Nikitin Y, Sandoya $E$, Kawecka-Jaszcz K, Ibsen $H$, Imai Y, Wang J, Staessen JA. Prognostic value of the morning blood pressure surge in 5645 subjects from 8 populations. Hypertension 2010; 55: 1040-1048.

26 Amici A, Cicconetti P, Sagrafoli C, Baratta A, Passador P, Pecci T, Tassan G, Verrusio W, Marigliano V, Cacciafesta M. Exaggerated morning blood pressure surge and cardiovascular events. A 5-year longitudinal study in normotensive and well-controlled hypertensive elderly. Arch Gerontol Geriatr 2009; 49: e105-e109.

27 Gosse P, Lasserre R, Minifie C, Lemetayer P, Clementy J. Blood pressure surge on rising. J Hypertens 2004; 22: 1113-1118.

28 Mancia G, Parati G. Importance of smooth and sustained blood pressure control in preventing cardiovascular morbidity and mortality. Blood Press Supp/ 2001: 26-32.

29 White WB. Cardiovascular risk and therapeutic intervention for the early morning surge in blood pressure and heart rate. Blood Press Monit 2001; 6: 63-72. 